\title{
Premier cas de Candida auris multirésistant déclaré au Canada
}

\author{
IS Schwartz ${ }^{1 *}$, GW Hammond ${ }^{1}$
}

\section{Résumé}

Candida auris, un champignon pathogène, est récemment devenu une menace pour la santé publique mondiale. Décrit pour la première fois au Japon en 2009, cet agent a depuis été signalé dans dix-sept pays sur cinq continents. La présente étude de cas décrit le premier cas de C. auris multirésistant déclaré au Canada.

En mai 2017, une personne âgée de 64 ans a été examinée pour une otite externe chronique. Ses antécédents médicaux comprenaient une hospitalisation récente en Inde pour une chirurgie buccale non urgente, compliquée par un abcès cérébral odontogénique. À son retour au Canada, la personne a été admise à l'hôpital pour drainage neurochirurgical de l'abcès cérébral et administration d'antibiotiques parentéraux. Au début de son hospitalisation, le patient a été identifié comme porteur d'entérobactéries résistantes aux carbapénèmes et a fait l'objet de précautions contre la transmission par contact. Une pose de drain transtympanique a également été réalisée afin d'évacuer du liquide clair de l'oreille et ainsi soigner une otite moyenne chronique. Le drain a été laissé en place pendant la durée de l'hospitalisation et également après le congé de l'hôpital pour admission dans un établissement de réadaptation après neurochirurgie. Lors d'une consultation externe de suivi, la mise en culture de prélèvements de l'écoulement auriculaire a révélé la présence de $C$. auris résistant au fluconazole et à l'amphotéricine B. II n'y a eu aucune réponse clinique à la thérapie antifongique ototopical; l'évaluation chirurgicale pour la prise en charge de l'otomastoïdite est en attente.

C. auris pourrait entraîner des infections nosocomiales. En effet, ce pathogène capable de persister dans les milieux hospitaliers présente un risque de transmission et peut être à l'origine de maladies invasives. Il est en outre difficile à identifier et souvent résistant aux médicaments antifongiques. L'application des recommandations de prévention et de contrôle des infections peut aider à prévenir la transmission nosocomiale. II est désormais prudent d'envisager le risque de présence de $C$. auris, en plus du risque connu lié à la présence d'autres microorganismes résistants aux antimicrobiens chez tout voyageur ayant été hospitalisé à l'extérieur du pays. Si le champignon est identifié, la communication avec les autorités locales de santé publique peut contribuer au suivi et à la gestion de cette maladie émergente. microorganismes résistants aux antimicrobiens chez tout voyageur ayant été hospitalisé à l'extérieur du pays. Si le champignon est identifié, la communication avec les autorités locales de santé publique peut contribuer au suivi et à la gestion de cette maladie émergente.

\section{Affiliations}

1 Division des maladies infectieuses, Faculté de médecine interne et de microbiologie médicale, Max Rady College of Medicine, Université du Manitoba, Winnipeg (Manitoba)

${ }^{*}$ Correspondence : ilan.steven. schwartz@gmail.com
Citation proposée : Schwartz IS, Hammond GW. Premier cas de Candida auris multirésistant au Canada. Relevé des maladies transmissibles au Canada. 2017;43(7/8):168-72. https://doi.org/10.14745/ccdr.v43i78a02f

\section{Cas}

En mai 2017, une personne âgée de 64 ans a été examinée pour une otite externe chronique. Le patient avait des problèmes d'oreilles récurrents depuis deux ans. Neuf mois avant la présentation, un omnipraticien a posé un diagnostic clinique d'otomycose en se fondant sur l'otalgie bilatérale et I'écoulement séreux épais d'un côté. L'application topique de pivalate de fluméthasone à $0,02 \%$ et de clioquinol à $1 \%$ a été prescrite pour une durée de huit semaines et a permis de résoudre l'écoulement. Deux mois plus tard, le patient s'est plaint d'une otalgie bilatérale sans écoulement et s'est vu prescrire de l'amoxicilline par voie orale par le même clinicien pour une otite moyenne aiguë présumée.

Les antécédents médicaux comprenaient une chirurgie buccale effectuée en Inde trois mois avant la présentation, intervention compliquée par un abcès cervical odontogène ayant entraîné une hospitalisation de 24 jours en Inde. Durant I'hospitalisation, un tomodensitogramme de l'os temporal a révélé une otite moyenne chronique avec mastoïdite et ostéomyélite de la mastoïde, de la paroi du rocher et de la paroi antérieure de la cavité de l'oreille moyenne d'un côté, et d'une mastoïdite peu sévère de l'autre côté. Le patient a reçu un traitement antibactérien et antituberculeux empirique, puis, après une amélioration clinique initiale, il est retourné au Canada et a consulté dans un hôpital communautaire pour obtenir d'autres soins. Un tomodensitogramme du cerveau a révélé un abcès frontal gauche persistant, et le patient a été transféré dans un hôpital de soins tertiaires pour examens complémentaires et traitement. Au début de son hospitalisation à l'hôpital tertiaire, le dépistage par prélèvements rectaux a permis d'identifier des 
entérobactéries résistantes aux carbapénèmes; le patient a donc fait l'objet de précautions contre la transmission par contact. Un aspirat de l'abcès cervical a été prélevé pour le diagnostic, et, malgré les résultats négatifs de la coloration de Gram et de la mise en culture bactériennes, mycobactériennes et fongiques, l'amplification et le séquençage de l'ARNr 16S ont permis d'isoler le streptocoque Streptococcus mitis. Le patient a reçu un traitement antibiotique parentéral pendant deux mois en tant que patient hospitalisé et durant un mois supplémentaire chez lui, avec une bonne réponse clinique et radiographique. Le patient avait également des antécédents d'emphysème, de diabète sucré latent et de cirrhose précoce asymptomatique d'étiologie indéterminée.

Peu de temps après son entrée à l'hôpital tertiaire au Canada pour le traitement de l'abcès cervical, le patient a également été traité par un otorhinolaryngologiste pour une otite moyenne chronique avec pose d'un drain transtympanique pour évacuer un épanchement clair. Le drainage a continué pendant les trois dernières semaines de son hospitalisation à l'hôpital de soins tertiaires, puis pendant son hospitalisation d'un mois pour réadaptation post-neurochirurgicale dans un troisième hôpital pour les complications découlant de l'abcès cérébral.

Lors d'une consultation externe de suivi, un prélèvement de l'écoulement auriculaire persistant a été envoyé pour une mise en culture bactérienne et fongique. Les analyses par spectrométrie de masse MALDI-TOF (Spectromètre Bruker MALDI, logiciel MBT Compass 4.1.70 pour recherche uniquement) ont révélé la présence du champignon

Candida auris. À l'aide de la méthode de microdilution en bouillon du CLSI et sur la base des seuils de concentration minimale inhibitrice proposés par les Centers for Diseases Control and Prevention (CDC) (1) des États-Unis, on a déterminé que l'échantillon de $C$. auris était résistant au fluconazole et à l'amphotéricine $\mathrm{B}$, probablement résistant au voriconazole et sensible à la micafungine (tableau 1). Le pathogène s'est également développé à partir de quatre prélèvements successifs de l'épanchement de la même oreille, réalisés sur une période de six semaines. Un isolat a été envoyé à un laboratoire de mycologie, où un essai de résistance médicamenteuse a été réalisé. L'analyse séquentielle du génome entier réalisée par le Laboratoire national de microbiologie (LNM) a permis d'établir une correspondance avec C. auris. Des analyses plus approfondies pour comparer l'isolat avec des souches mondiales sont en cours.

Tableau 1 : Résumé des tests de sensibilité de l'isolat de Candida auris provenant du premier cas déclaré au Canada

\begin{tabular}{|l|l|l|l|l|}
\hline \multicolumn{1}{|c|}{ Classe } & Médicament & $\begin{array}{c}\text { Seuils } \\
\text { de CMI }\end{array}$ & $\begin{array}{c}\text { Résultats de } \\
\text { I'isolat de } \\
\text { C. auris }\end{array}$ & $\begin{array}{c}\text { Interpréta- } \\
\text { tion }\end{array}$ \\
\hline \multirow{2}{*}{ Triazoles } & Fluconazole & $\geq 32 \mu \mathrm{g} / \mathrm{mL}$ & $128 \mu \mathrm{g} / \mathrm{mL}$ & Résistant \\
\cline { 2 - 5 } & Voriconazole & s.0. ${ }^{2}$ & s.o. ${ }^{2}$ & $\begin{array}{l}\text { Résistance } \\
\text { probable }{ }^{2}\end{array}$ \\
\hline Polyènes & Amphotéricine B & $\geq 2 \mu \mathrm{g} / \mathrm{mL}$ & $2 \mu \mathrm{g} / \mathrm{mL}$ & Résistant \\
\hline Échinocandines & Micafungine & $\geq 4 \mu \mathrm{g} / \mathrm{mL}$ & $0,5 \mu \mathrm{g} / \mathrm{mL}$ & Sensible \\
\hline
\end{tabular}

Abréviations : $\mathrm{CMI}$, concentration minimale inhibitrice, s.o., sans objet car non disponible 1 Seuils (CMI) pour C. auris définis par les Centers for Disease Control and Prevention des ÉtatsUnis(1)

${ }^{2}$ En attendant qu'un seuil de CMI soit défini les Centers for Disease Control and Prevention des États-Unis suggèrent d'utiliser la sensibilité au fluconazole comme substitut pour l'évaluation de la sensibilité au triazole de deuxième génération, en notant toutefois que des isolats résistants au fluconazole peuvent parfois répondre à d'autres triazoles
Une réévaluation en consultation externe avec un otorhinolaryngologiste a mis en évidence une myringite et une otite externe. Le conduit auditif était tapissé de dépôts blancs et humides, qui ont été enlevés. Le drain transtympanique posé antérieurement permettait l'évacuation d'un liquide clair et a été laissé en place. Le patient a de nouveau reçu un traitement topique sous forme de gouttes de pivalate de fluméthasone à $0,02 \%$ et de clioquinol à $1 \%$, mais sans amélioration après deux semaines. Le patient a été orienté vers un neurotologue pour évaluation chirurgicale. La résolution de cas d'otite externe et de la mastoïdite chronique est en cours au moment de la rédaction de la présente étude.

\section{Discussion}

II s'agit du premier cas de C. auris multirésistant recensé au Canada. Décrit pour la première fois au Japon en 2009(3), ce pathogène a depuis été signalé dans dix-sept pays sur cinq continents (2,4). Avant 2009 (5), seuls quatre isolats de C. auris avaient été identifiés rétrospectivement parmi les 15271 isolats d'un registre mondial de la septicémie à candida, indiquant une dispersion globale rapide. C. auris a déjà envahi durablement certains milieux de soins de santé. Dans les pays en développement, le risque de transmission de C. auris est aggravé par le manque de moyens pour l'identification des champignons, les tests de sensibilité antifongiques et la prévention et le contrôle des infections. En Inde, par exemple, C. auris représente déjà $5 \%$ des isolats impliqués dans les septicémies à candida au sein des unités de soins intensifs du pays (6) et jusqu'à $30 \%$ dans certains centres (7).

Plusieurs caractéristiques de C. auris rendent l'infection difficile à traiter et renforcent les inquiétudes quant à la transmission nosocomiale : il peut persister chez les patients et en milieu hospitalier; il est difficile à identifier, il peut engendrer une maladie invasive et les options de traitement sont limitées $(1,4,7)$. En effet, des épidémies récurrentes associées aux soins de santé ont été signalées au Royaume-Uni et aux États-Unis $(9,10)$, et les études génétiques ont mis en cause des souches (clonales) très proches, ce qui suggère une transmission horizontale efficace $(5,7,9-10)$.

\section{C. auris peut persister dans les environnements hospitaliers}

La ténacité de $C$. auris a été démontrée lors des enquêtes sur les éclosions, au cours desquelles l'organisme a pu être isolé de la peau de patients (jusqu'à trois mois après l'infection) et des environnements de patients $(9,14)$. La capacité de $C$. auris à adhérer aux surfaces polymériques et à former des biofilms pourrait contribuer à la difficulté à éradiquer cet organisme de l'environnement (15).

\section{C. auris est difficile à détecter}

L'identification correcte de C. auris peut être difficile pour les laboratoires de microbiologie clinique, ce qui pourrait retarder la mise en place des procédures de $\mathrm{PCl}$ appropriées. C. auris peut être mal identifié par les systèmes d'identification commerciaux tels que Vitek-2 (comme C. haemulonii ou C. famata) et API-20C (comme Rhodotorula glutinis, C. sake ou Saccharomyces cerevisiae) (16). C. auris peut être correctement identifié par MALDI-TOF à l'aide des bases de données « pour 
fins de recherche uniquement » et par séquençage de l'espaceur interne transcrit et des domaines D1-D2 (16). Il existe aussi des limitations avec certaines méthodes de test de sensibilité antifongique (16); par exemple, Vitek-2 pourrait donner des CMI faussement élevées pour l'amphotéricine $B$ et la méthode de microdilution en milieu liquide pourrait donner des $\mathrm{CMI}$ faussement élevées pour la caspofungine (16).

\section{C. auris peut provoquer une maladie invasive}

Bien que les premiers cas signalés de $C$. auris touchaient des patients atteints d'otomycose chronique $(3,11)$, la plupart des cas subséquents étaient liés aux soins de santé et concernaient une septicémie à candida ou d'autres infections invasives (2). Une septicémie à candida persistante (durant jusqu'à trois semaines après l'instauration des antifongiques) et des taux de mortalité élevés ont été observés (7). Un modèle murin de septicémie à candida a laissé envisager que $C$. auris était pratiquement aussi virulent que $C$. albicans, la cause prédominante de candidose invasive dans le monde (12); les facteurs de virulence précis sont toujours à l'étude, bien que certains semblent liés à la souche (13).

\section{Les options thérapeutiques sont limitées}

La sensibilité réduite aux antifongiques parmi les isolats est une caractéristique universelle de C. auris. La résistance au fluconazole dépasse $90 \%$ (5) et la sensibilité réduite au voriconazole pourrait être proche de 50 \% (2). On ne possède pas de seuils de $\mathrm{CMI}$ pour les plus récents triazoles, le posaconazole et l'isavuconazole, mais les faibles CMI laissent envisager qu'ils pourraient agir contre C. auris (2). Jusqu'à $35 \%$ des isolats présentent une résistance à l'amphotéricine $\mathrm{B}(5)$ et la résistance à l'échinocandine a été signalée dans 2 à $8 \%$ des isolats $(2,5)$. Une résistance aux trois grandes classes d'antifongiques a été signalée dans $4 \%$ des isolats (5).

La prise en charge optimale de la maladie à C. auris n'a pas encore été établie (2). En cas de maladie invasive, un traitement antifongique systémique devrait être guidé par les résultats des tests de sensibilité. La prise en charge de l'otomycose et de I'otomastoidite provoquée par $C$. auris est encore moins claire. Il n'a pas été publié d'évaluations de l'efficacité des agents ototopiques contre $C$. auris, et les options pour les isolats multirésistants, comme dans ce cas, sont limitées.

\section{La prévention et le contrôle des infections peuvent limiter la propagation}

Les mesures de prévention et de contrôle des infections peuvent limiter efficacement la propagation de C. auris. Les recommandations préliminaires de $\mathrm{PCl}$ des Centers for Disease Control and Prevention des États-Unis comprennent les chambres individuelles et les précautions contre la transmission par contact pour les patients colonisés ou infectés, la répétition périodique de la procédure d'écouvillonnage à la recherche de colonisation ainsi que le nettoyage quotidien et final des surfaces avec un désinfectant efficace contre les spores de Clostridium difficile (voir encadré) (10).
Résumé des recommandations préliminaires des Centers for Disease Control and Prevention des États-Unis pour Candida auris dans les établissements de santé (10)

\section{Appliquer des précautions standard et des précautions} contre la transmission par contact

Les patients dans les hôpitaux de soins de courte durée et les maisons de soins infirmiers doivent être placés en chambre individuelle et être soumis à des précautions standard et à des précautions contre la transmission par contact. Les précautions contre la transmission par contact doivent être appliquées tant que le patient est colonisé par C. auris.

\section{Utiliser un désinfectant actif contre les spores de Clostridium difficile}

Les établissements de santé qui ont des patients infectés ou colonisés par $C$. auris doivent s'assurer d'effectuer un nettoyage et une désinfection quotidiens et finaux des chambres de ces patients avec un désinfectant hospitalier efficace contre les spores de Clostridium difficile.

\section{Effectuer des réévaluations périodiques}

Des réévaluations périodiques à la recherche de colonisation par C. auris (tous les 1 à 3 mois) peuvent aider à établir la durée des mesures de contrôle des infections. Les évaluations de la colonisation devraient comprendre I'analyse, au minimum, d'écouvillons des aisselles et de l'aine et pourraient aussi comprendre des sites ayant montré la présence de $C$. auris lors de cultures antérieures.

\section{Aviser lors du transfert}

Lorsque les patients sont transférés à d'autres établissements de santé, les établissements de santé qui reçoivent les patients doivent être avisés de l'infection ou de la colonisation par $C$. auris de même que du niveau de précautions recommandé.

Documenter deux tests négatifs pour $C$. auris avant d'arrêter les procédures de contrôle des infections

Deux évaluations ou plus effectuées à une semaine $d$ 'intervalle donnant des résultats négatifs sont nécessaires avant d'arrêter les précautions de contrôle des infections. Le patient ou le résident ne doit pas recevoir de médicaments antifongiques actifs contre $C$. auris au moment de ces évaluations (attendre une semaine).

\section{Conclusion}

Ce patient est actuellement cliniquement stable et attend une évaluation chirurgicale. Ce patient est actuellement cliniquement stable et attend une évaluation chirurgicale. Les pratiques exemplaires en matière de $\mathrm{PCl}$ sont actuellement mises en place dans les trois hôpitaux où l'on a prodigué des soins à ce patient; les autorités de santé publique locales ont été avisées, et un suivi des mesures de $\mathrm{PCl}$ est en cours à l'échelle régionale. Heureusement, dans le cadre du traitement de l'abcès du cerveau associé à l'hospitalisation du patient à l'étranger, le dépistage de routine des organismes résistants aux antimicrobiens a permis de dépister des entérobactéries 
productrices de carbapénèmases; des précautions contre la transmission par contact ont donc été prises pour ce patient.

La source de C. auris n'a pas encore été établie, mais une source pourrait avoir été liée à l'hospitalisation en Inde, où $C$. auris est connu pour être endémique $(6,17)$. En raison de la chronicité des symptômes auriculaires et le diagnostic antérieur (non confirmé) d'otomycose, l'infection pourrait avoir précédé le tout dernier voyage du patient; toutefois, le patient s'était rendu en Inde avant la survenue des symptômes auriculaires aussi.

Tout comme les autres organismes résistants aux antimicrobiens (18), les cliniciens observent vraisemblablement de plus en plus de cas d'infection ou de colonisation par C. auris associés au voyage. Aux États-Unis, deux cas d'infection à C. auris ont été observés chez des voyageurs rentrant au pays ayant été hospitalisés à l'étranger $(10,14)$. En outre, au cours d'une éclosion liée aux hôpitaux touchant plusieurs États aux États-Unis, les isolats C. auris provenant de New York et New Jersey étaient étroitement liés les uns avec les autres et étaient semblables aux isolats sud-asiatiques (10); cela pourrait laisser envisager l'introduction de $C$. auris acquis à l'étranger dans les établissements de santé dans ces États. Quiconque ayant été hospitalisé lors d'un séjour à l'étranger est à risque accru relativement aux organismes résistants aux antimicrobiens, y compris $C$. auris; des précautions contre la transmission par contact et des tests pourraient être indiqués. Cette approche pourrait aider à limiter la transmission nosocomiale de $C$. auris au sein des établissements de santé canadiens et entre ceux-ci. Alerter les autorités de santé publique locales peut également appuyer les efforts de surveillance et de gestion de cette maladie émergente.

\section{Déclarations des auteurs}

ISS - Conceptualisation, collecte des données, rédaction version initiale, révision et édition

GWH - Conceptualisation, recherche, collecte des données, rédaction - révision, édition et supervision

\section{Conflit d'intérêt}

Aucun.

\section{Remerciements}

Les auteurs remercient chaleureusement le patient ayant donné son consentement écrit à la publication de ce rapport de cas, le $D^{r}$ Arnold Frohlich pour son opinion clinique d'expert et pour la révision du manuscrit, les microbiologistes et techniciens des Diagnostic Services Manitoba, Cadham Provincial Laboratory et le Laboratoire national de microbiologie. Nous remercions les $D^{\text {rs }}$ Eric Bow et Kelly MacDonald pour leurs discussions utiles sur la prise en charge des cas. Les auteurs souhaitent aussi remercier l'équipe rédactionnelle du RMTC et les réviseurs anonymes pour les évaluations expresses de cette communication rapide.

\section{Références}

1. Centers for Disease Control and Prevention (CDC). Candida auris Interim Recommendations for Healthcare Facilities and Laboratories [Internet]. [consulté le 23 juin 2017]. Disponible à l'adresse: https://www.cdc.gov/fungal/diseases/ candidiasis/recommendations.html.

2. Chowdhary A, Sharma C, Meis JF. Candida auris: A rapidly emerging cause of hospital-acquired multidrugresistant fungal infections globally. PLoS Pathog 2017 May;13(5):e1006290. DOI: http://dx.doi.org/10.1371/ journal.ppat.1006290. PubMed (https://www.ncbi.nlm.nih. gov/entrez/query.fcgi?cmd=Retrieve \&db=PubMed\&lis t_uids=28542486\&dopt=Abstract).

3. Satoh K, Makimura K, Hasumi Y, Nishiyama Y, Uchida K, Yamaguchi $H$. Candida auris sp. nov., a novel ascomycetous yeast isolated from the external ear canal of an inpatient in a Japanese hospital. Microbiol Immunol 2009 Jan;53(1):41-4. DOI: http://dx.doi.org/10.1111/j.13480421.2008.00083.x. PubMed (https://www.ncbi.nlm.nih. gov/entrez/query.fcgi?cmd=Retrieve \&db=PubMed\&lis t_uids=19161556\&dopt=Abstract).

4. Al-Siyabi T, Al Busaidi I, Balkhair A, Al-Muharrmi Z, Al-Salti $M, A l^{\prime} A d a w i$ B. First report of Candida auris in Oman: clinical and microbiological description of five candidemia cases. J Infect 2017 Jun;4453(17)1-3:S0163-4453(17)30164-0. 10.1016/j.jinf.2017.05.016. PubMed (https://www.ncbi.nlm. nih.gov/entrez/query.fcgi?cmd=Retrieve\&db=PubMed\&li st_uids=28579303\&dopt=Abstract).

5. Lockhart SR, Etienne KA, Vallabhaneni S, Faroogi J, Chowdhary A, Govender NP et al. Simultaneous Emergence of Multidrug-Resistant Candida auris on 3 Continents Confirmed by Whole-Genome Sequencing and Epidemiological Analyses. Clin Infect Dis 2017 Jan;64(2):13440. DOI: http://dx.doi.org/10.1093/cid/ciw691. PubMed (https://www.ncbi.nlm.nih.gov/entrez/query.fcgi?cmd=Retrie $v e \& d b=$ PubMed\&list_uids=27988485\&dopt=Abstract).

6. Rudramurthy SM, Chakrabarti A, Paul RA, Sood P, Kaur $\mathrm{H}$, Capoor MR et al. Candida auris candidaemia in Indian ICUs: analysis of risk factors. J Antimicrob Chemother 2017 Jun;72(6):1794-801. DOI: http://dx.doi.org/10.1093/ jac/dkx034. PubMed (https://www.ncbi.nlm.nih.gov/ entrez/query.fcgi?cmd=Retrieve\&db=PubMed\&list_ uids=28333181\&dopt=Abstract).

7. Chowdhary A, Sharma C, Duggal S, Agarwal K, Prakash A, Singh PK et al. New clonal strain of Candida auris, Delhi, India. Emerg Infect Dis [Internet]. Centers for Disease Control and Prevention; 2013 Oct [consulté le 27 juin 2017];19(10):1670-3. Disponible à l'adresse : http://www. ncbi.nlm.nih.gov/pubmed/24048006.

8. Clancy CJ, Nguyen MH. Emergence of Candida auris : An International Call to Arms. Clin Infect Dis [Internet]. 2017 Jan 15 [consulté le 22 juin 2017];64(2):141-3. Disponible à l'adresse : http://www.ncbi.nlm.nih.gov/pubmed/27989986.

9. Schelenz S, Hagen F, Rhodes JL, Abdolrasouli A, Chowdhary A, Hall A et al. First hospital outbreak of the globally emerging Candida auris in a European hospital. Antimicrob Resist Infect Control 2016 Oct;5(1):35-42. DOI: http://dx.doi. 
Am J Transplant 2017 Jan;17(1):296-9. DOI: http://dx.doi. org/10.1111/ajt.14121. PubMed (https://www.ncbi.nlm. nih.gov/entrez/query.fcgi?cmd=Retrieve\&db=PubMed\&li st_uids=28029734\&dopt=Abstract).

15. Sherry L, Ramage G, Kean R, Borman A, Johnson EM, Richardson MD et al. Biofilm-Forming Capability of Highly Virulent, Multidrug-Resistant Candida auris. Emerg Infect Dis 2017 Feb;23(2):328-31. DOI: http://dx.doi.org/10.3201/ eid2302.161320. PubMed (https://www.ncbi.nlm.nih. gov/entrez/query.fcgi?cmd=Retrieve\&db=PubMed\&lis t_uids=28098553\&dopt=Abstract).

16. Kathuria S, Singh PK, Sharma C, Prakash A, Masih A, Kumar A et al. Multidrug-Resistant Candida auris Misidentified as Candida haemulonii: Characterization by MatrixAssisted Laser Desorption Ionization-Time of Flight Mass Spectrometry and DNA Sequencing and Its Antifungal Susceptibility Profile Variability by Vitek 2, CLSI Broth Microdilution, and Etest Method. J Clin Microbiol 2015 Jun;53(6):1823-30. DOI: http://dx.doi.org/10.1128/ JCM.00367-15. PubMed (https://www.ncbi.nlm.nih. gov/entrez/query.fcgi?cmd=Retrieve \&db=PubMed\&lis t_uids=25809970\&dopt=Abstract).

17. Chowdhary A, Anil Kumar V, Sharma C, Prakash A, Agarwal $\mathrm{K}, \mathrm{Babu} \mathrm{R}$ et al. Multidrug-resistant endemic clonal strain of Candida auris in India. Eur J Clin Microbiol Infect Dis 2014 Jun;33(6):919-26. DOI: http://dx.doi.org/10.1007/ s10096-013-2027-1. PubMed (https://www.ncbi.nlm.nih. gov/entrez/query.fcgi?cmd=Retrieve\&db=PubMed\&lis t_uids=24357342\&dopt=Abstract).

18. MacFadden DR, Bogoch II, Brownstein JS, Daneman N, Fisman D, German M et al. A passage from India: Association between air traffic and reported cases of New Delhi Metallobeta-lactamase 1 from 2007 to 2012. Travel Med Infect Dis [Internet]. 2015 Jul [consulté le 24 juin 2017];13(4):295-9. Disponible à l'adresse : http://linkinghub.elsevier.com/ retrieve/pii/S1477893915001040. 